\title{
Narrow-band imaging with magnifying endoscopy for Peyer's patches is useful in predicting the recurrence of remissive patients with ulcerative colitis
}

\author{
Satoshi Hiyama ${ }^{1,2}$, Hideki Iijima ${ }^{1}$, Syoichiro Kawai ${ }^{1}$, Akira Mukai ${ }^{3}$, Eri Shiraishi ${ }^{3}$, Shuko Iwatani ${ }^{1}$, \\ Toshio Yamaguchi ${ }^{1}$, Manabu Araki ${ }^{1}$, Yoshito Hayashi ${ }^{1}$, Shinichiro Shinzaki ${ }^{1}$, Tsunekazu Mizushima ${ }^{2,4}$, \\ Masahiko Tsujii ${ }^{5}$, Tetsuo Takehara ${ }^{1}$ \\ Departments of ${ }^{1}$ Gastroenterology and Hepatology and ${ }^{2}$ Therapeutics for Inflammatory Bowel Diseases, Osaka University Graduate School of \\ Medicine, Osaka, ${ }^{3}$ Department of Gastroenterology, Sumitomo Hospital, Osaka, ${ }^{4}$ Department of Gastroenterological Surgery, Osaka University \\ Graduate School of Medicine, Osaka, ${ }^{5}$ Department of Gastroenterology, Osaka Rosai Hospital, Osaka, Japan
}

\begin{abstract}
Background/Aims: Peyer's patches (PPs) are aggregates of lymphoid follicles that are mainly located in the distal ileum; they play a major role in mucosal immunity. We recently reported that patients with ulcerative colitis (UC) have alterations in PPs that can be detected using narrow-band imaging with magnifying endoscopy (NBI-ME). However, the usefulness of NBI-ME in UC treatment as a whole is still unknown. Methods: We collected NBI-ME images of PPs from 67 UC patients who had undergone ileocolonoscopy. We evaluated changes in the villi using the "villi index," which is based on three categories: irregular formation, hyperemia, and altered vascular network pattern. The patients were divided into two groups on the basis of villi index: low (L)- and high (H)-types. We then determined the correlation between morphological alteration of the PPs and various clinical characteristics. In 52 patients who were in clinical remission, we also analyzed the correlation between NBI-ME findings of PPs and clinical recurrence. Results: The time to clinical recurrence was significantly shorter in remissive UC patients with $\mathrm{H}-$ type PPs than in those with L-type PPs $(P<0.01)$. Moreover, PP alterations were not correlated with age, sex, disease duration, clinical activity, endoscopic score, or extent of disease involvement. Multivariate analysis revealed that the existence of H-type PPs was an independent risk factor for clinical recurrence (hazard ratio, $3.3 ; P<0.01$ ). Conclusions: UC patients with morphological alterations in PPs were at high risk of clinical relapse. Therefore, to predict the clinical course of UC, it may be useful to evaluate NBI-ME images of PPs. (Intest Res 2016;14:314-321)
\end{abstract}

Key Words: Colitis, ulcerative; Peyer's patch; Narrow-band imaging

Received April 3, 2016. Revised May 30, 2016.

Accepted June 7, 2016.

Correspondence to Hideki lijima, Department of Gastroenterology and Hepatology, Osaka University Graduate School of Medicine, 2-2 Yamadaoka, Suita, Osaka 565-0871, Japan. Tel: +81-6-6879-3621, Fax: +81-6-68793629, E-mail: hiijima@gh.med.osaka-u.ac.jp

Financial support: This work was supported by a Grant-in-Aid for Scientific Research (C) from the Japan Society for the Promotion of Science (26460969), Grant-in-Aid for Research Activity Start-up from the Japan Society for the Promotion of Science (26893136), and Osaka Medical Research Foundation for Intractable Diseases. Conflict of interest: None.

\section{INTRODUCTION}

Peyer's patches (PPs) are aggregates of lymphoid follicles, almost half of which are located in the distal $25 \mathrm{~cm}$ of the ileum. As gut-associated lymphoid tissue (GALT), PPs play a major role in activating immune responses against infection, as well as in maintaining oral tolerance against gut commensal microbes and food antigens. ${ }^{1,2}$ Indeed, PPs are considered to be the major inductive site for the mucosal immune responses of GALT, because luminal antigens are taken up through M (microfold)-cells, which are located within PPs. ${ }^{1}$

\footnotetext{
๑ Copyright 2016. Korean Association for the Study of Intestinal Diseases. All rights reserved.

This is an Open Access article distributed under the terms of the Creative Commons Attribution Non-Commercial License (http://creativecommons.org/licenses/by-nc/4.0)

which permits unrestricted non-commercial use, distribution, and reproduction in any medium, provided the original work is properly cited.
} 
Taken together, this suggests that PPs are involved in the pathogenesis of gut immune diseases, such as IBD.

Specifically, IBD comprises two categories of diseases, UC and $\mathrm{CD}$, both of which are intractable diseases of the digestive tract. Because gut flora or food antigens are involved in the pathogenesis of IBD, ${ }^{3}$ and because the uptake of luminal antigens occurs within PPs, it has been speculated that immune responses within PPs play an important role in the onset and clinical course of UC. In CD, aphthoid lesions within PPs have been reported as the initial inflammatory lesions. ${ }^{4}$ Nonetheless, the role of PPs in UC is not fully understood.

Colonoscopy is widely performed in patients with UC; it is useful in diagnosis, treatment decisions, and cancer surveillance. ${ }^{5}$ However, little attention has been paid to PPs, because they cannot be readily seen using conventional endoscopy. On this note, we recently reported that PPs can be visualized using narrow-band imaging with magnifying endoscopy (NBI-ME); microstructurally, they appear as aggregates of domed structures, surrounded by villi. ${ }^{6}$ We also reported that patients with IBD have structural and vascular alterations within the dome area, as well as in the surrounding villi. ${ }^{6}$ However, the clinical significance of these alterations in patients with UC has not been well evaluated. In the present study, we aimed to evaluate the association between NBIME images of PPs and clinical course in patients with UC.

\section{METHODS}

\section{Patients}

Between April 2009 and March 2013, we enrolled consecutive patients with UC who had undergone total ileocolonoscopy, and whose terminal ileal lesions were observed by NBI-ME in the Department of Gastroenterology and Hepatology, Osaka University Hospital. UC was diagnosed on the basis of endoscopic, radiological, histological, and clinical criteria. ${ }^{7,8}$ Clinical activity was assessed using a partial Mayo score, ${ }^{9}$ and endoscopic activity was evaluated using a Mayo endoscopic subscore. ${ }^{9}$ Clinical recurrence was defined as a partial Mayo score $\geq 3$. The exclusion criteria were as follows: (1) history of colectomy; (2) current total parenteral nutrition; (3) current diagnosis of indeterminate colitis; and (4) history of malignancy. The study protocol was approved by the Ethics Committee of the Osaka University Graduate School of Medicine, and written informed consent was obtained from each participant.

\section{Endoscopic Procedure}

Colonoscopic examinations were performed using a magnifying video colonoscope system (CF-H260AZI ${ }^{\mathrm{TM}}$; Olympus, Tokyo, Japan) with a video processor system (EVIS 260 Lucera Spectrum $^{\mathrm{TM}}$; Olympus), while PPs in the terminal ileum, within $10 \mathrm{~cm}$ from Bauhin's valve, were observed using NBI-ME. In accordance with previous literature, we defined PPs as regions comprising more than five domes. ${ }^{10}$ When several PPs were observed in the terminal ileum, we evaluated the PP that was located closest to the anus.

\section{Evaluation of PPs Using NBI-ME}

The images of PPs, obtained using NBI-ME, were evaluated on the basis of three characteristics of the surrounding villi, as previously reported: (1) irregular formation; (2) hyperemia; and (3) altered vascular network pattern (Supplementary Fig. 1). ${ }^{6}$ The images were reviewed, in a blinded manner, by three independent gastroenterologists. A point was added for each category when at least two reviewers agreed, and the "villi index" was calculated as the sum of the applied categories (score, $0-3$ ). The patients were divided into two groups on the basis of the villi index: the low (L)-type group included patients with a score of 0 or 1 , while patients in the high (H)-type group had a score of 2 or 3.

\section{Statistical Analysis}

The overall differences between groups were determined using the Kruskal-Wallis test. The Mann-Whitney $U$ test or the chi-square test was used to compare baseline characteristics between patients with L-type and H-type PPs. Time-torelapse curves after the colonoscopy were evaluated using the Kaplan-Meier method, and statistical significance was determined using the log-rank test. Prognostic factors affecting relapse were initially examined using a univariate Cox proportional hazards model; this was followed by multivariate analysis to adjust for potential confounders. A $P$-value of less than 0.05 was considered statistically significant.

\section{RESULTS}

\section{Clinical Characteristics of the Patients}

Sixty-seven patients with UC who had undergone ileocolonoscopy were enrolled between April 2009 and March 2013. The patients' characteristics are shown in Table 1 . The 
Table 1. Patient Characteristics

\begin{tabular}{|c|c|c|c|c|}
\hline Variable & Total $(n=67)$ & L-type $(n=35)$ & H-type $(n=32)$ & $P$-value \\
\hline Age at colonoscopy (yr) & $44.2(17-75)$ & $45.7(21-68)$ & $42.5(17-75)$ & 0.3200 \\
\hline Age at diagnosis (yr) & $34.5(14-71)$ & $36.4(16-68)$ & $32.5(14-71)$ & 0.0800 \\
\hline Female/male & $28 / 39$ & $15 / 20$ & $13 / 19$ & 0.8500 \\
\hline Disease duration (yr) & $9.5(0-36)$ & $9.3(0-28)$ & $9.8(0-36)$ & 0.8000 \\
\hline Disease extent (pancolitis/left-sided colitis/proctitis) & $29 / 22 / 16$ & 13/13/9 & $16 / 9 / 7$ & 0.3900 \\
\hline Partial Mayo score $(\leq 2 / \geq 3)$ & $52 / 15$ & $29 / 6$ & $23 / 9$ & 0.2800 \\
\hline Mayo endoscopic subscore (0/1/2/3) & $23 / 30 / 10 / 4$ & $10 / 17 / 7 / 1$ & $13 / 13 / 3 / 3$ & 0.6500 \\
\hline Inflammation in terminal ileum (present/absent) & $4 / 63$ & $1 / 34$ & $3 / 29$ & 0.2600 \\
\hline Reddish peri-appendiceal mucosa (present/absent) & $14 / 53$ & $2 / 33$ & $12 / 20$ & 0.0014 \\
\hline \multicolumn{5}{|l|}{ Concomitant medication } \\
\hline Aminosalicylates & $54(81.0)$ & $28(80.0)$ & $26(81.3)$ & 0.9000 \\
\hline Oral corticosteroids & $6(9.0)$ & $2(5.7)$ & $4(12.5)$ & 0.3300 \\
\hline Immunomodulator & $8(11.9)$ & $3(8.6)$ & $5(15.6)$ & 0.3700 \\
\hline Anti-TNF therapy & $1(1.5)$ & 0 & $1(3.1)$ & 0.2900 \\
\hline No medication & $13(19.4)$ & $7(20.0)$ & $6(18.8)$ & 0.9000 \\
\hline
\end{tabular}

Values are presented as average (range) or number (\%).

L-type, low-type; H-type, high-type; TNF, tumor necrosis factor.

majority of the patients $(78 \%, 52 / 67)$ were in clinical remission (partial Mayo score $\leq 2$ ), and the Mayo endoscopic subscore was 0 in 23 patients (34\%), 1 in 30 patients (45\%), 2 in 10 patients (15\%), and 3 in four patients (6\%).

\section{NBI-ME Images of PPs and Clinical Characteristics}

Under NBI-ME, PPs manifest as areas with multiple domes surrounded by villi (Fig. 1). The villi indices of the PPs, ascribed on the basis of NBI-ME, ranged from 0 to 3 in patients with UC. There was no relationship between the Mayo endoscopic subscore and the villi index (Fig. 2A). We divided the PPs into L-type (villi index, 0 or 1) and H-type (villi index, 2 or 3) PPs. Representative images of L- and H-type PPs are shown in Fig. 2B and C, respectively. Thirty-five patients (52\%) had L-type PPs, while 32 (48\%) had H-type PPs (Fig. 2D). When observed under NBI without magnification, Ltype PPs had a low frequency of brownish lesions (Supplementary Fig. 2A); conversely, H-type PPs appeared as faint areas with irregular, brown spots inside the PPs (Supplementary Fig. 2B). There were no significant associations between the types of PPs (L- or H-type) and the following clinical characteristics: age at ileocolonoscopy, age at diagnosis, gender, and disease duration. In addition, the types of PPs were not associated with the extent of disease involvement, clini-

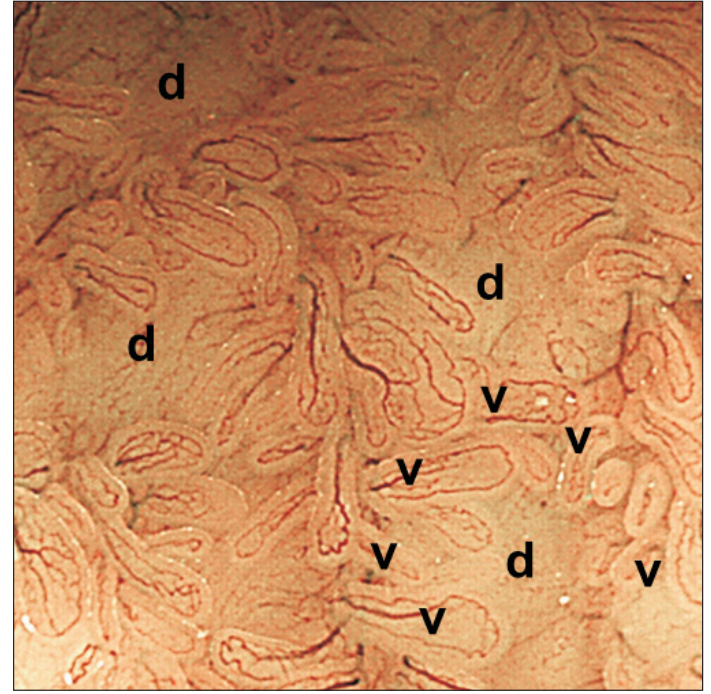

Fig. 1. Narrow-band imaging with magnifying endoscopy allowed us to clearly observe Peyer's patches: aggregates of multiple domes (d) surrounded by villi (v) with semi-circular vessels.

cal activity, endoscopic degree of inflammation, or concomitant medications. Notably, patients with H-type PPs had a significantly higher rate of reddish peri-appendiceal mucosa (Fig. 2E) than patients with L-type PPs (Table 1). There was no difference between patients with L- and H-type PPs with 

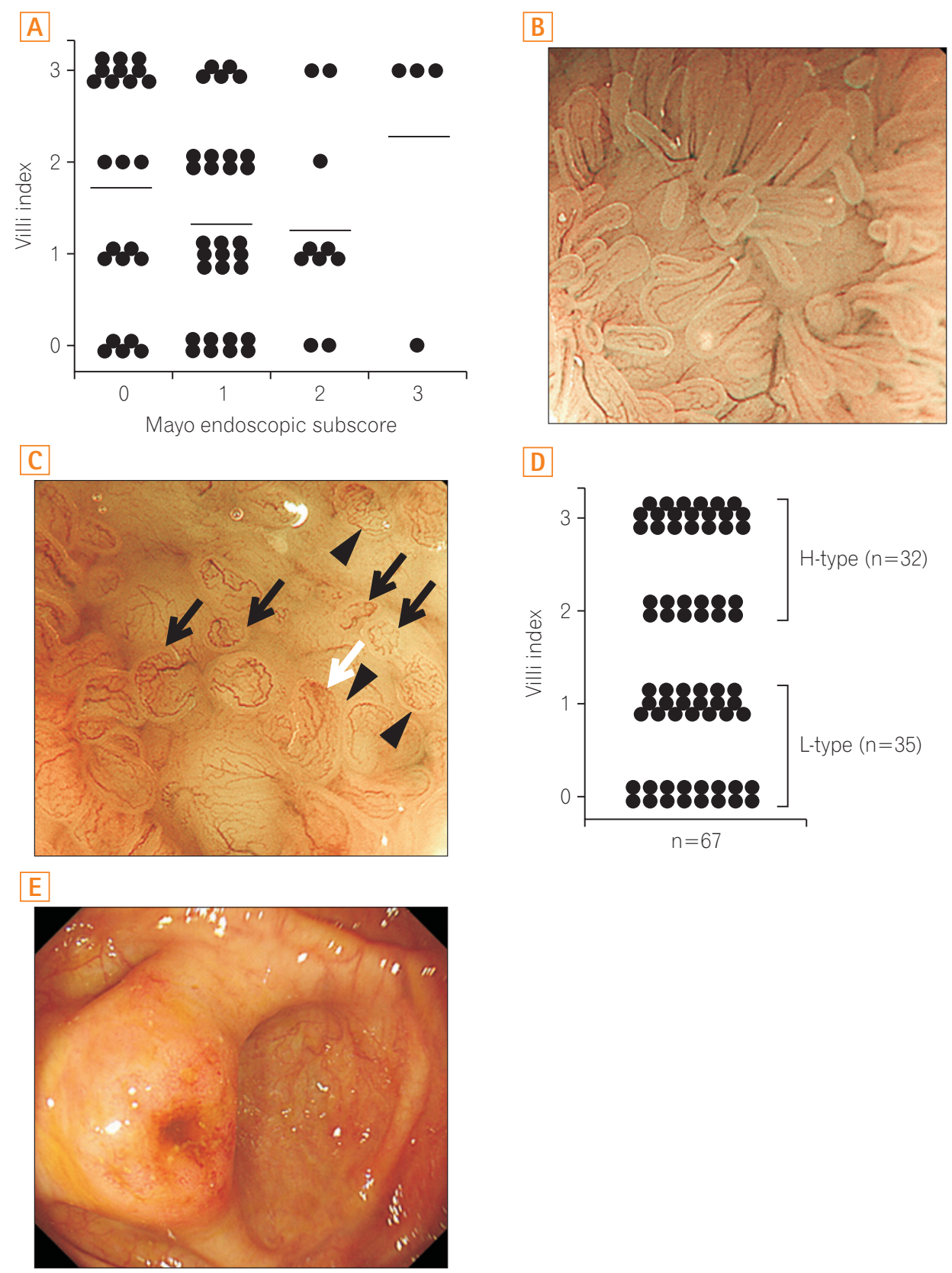

Fig. 2. (A) Scatterplot of the villi index against Mayo endoscopic subscore at the colonoscopy. No correlation was seen between the villi index and the Mayo endoscopic subscore. Bars represent the mean. Representative images of L-type (B) and $\mathrm{H}$ type (C) Peyer's patches (PPs) (black arrows, irregular formation; white arrow, hyperemia; arrowheads, altered vascular network pattern). (D) Evaluation of PPs by narrowband imaging with magnifying endoscopy in UC patients using the "villi index." Thirtyfive patients showed L-type PPs (villi index, 0 or 1), while 32 showed H-type PPs (villi index, 2 or 3). (E) A representative image of the reddish peri-appendiceal mucosa in patients with UC. H-type, high-type; Ltype, low-type.

regard to either the extent of disease involvement or inflammation in the terminal ileum (Table 1).

\section{Clinical Recurrence Rate in Remissive UC Patients with $L$ - and $H$-type PPs}

Next, in 52 remissive patients with UC, we investigated the association between the type of villi within the PPs and clinical recurrence (partial Mayo score $\leq 2$ ). Twenty-nine patients had L-type PPs and 23 had H-type PPs. Among these patients, the Mayo endoscopic subscore at the time of colonos- copy was 0 in 23 patients, 1 in 26 patients, and 2 in three patients. None of the patients had a Mayo endoscopic subscore of 3. The characteristics of the patients are shown in Table 2. The mean observation period was 24 months (range, 1-62 months). Among the remissive patients, the rate of reddish peri-appendiceal mucosa was significantly higher in patients with H-type PPs than in patients with L-type PPs $(P=0.014)$ (Table 2). Otherwise, there were no significant differences in clinical background between the two groups.

Among these 52 patients, 21 experienced relapse during the observation period. The cumulative clinical recurrence 
Table 2. Characteristics of Patients in Clinical Remission

\begin{tabular}{|c|c|c|c|}
\hline Variable & L-type $(n=29)$ & H-type $(n=23)$ & $P$-value \\
\hline Age at colonoscopy $(<40 / \geq 40 \mathrm{yr})$ & $11 / 18$ & $13 / 10$ & 0.140 \\
\hline Age at diagnosis $(<40 / \geq 40 \mathrm{yr})$ & $17 / 12$ & $16 / 7$ & 0.300 \\
\hline Female/male & $13 / 16$ & $13 / 10$ & 0.290 \\
\hline Disease duration $(<10 / \geq 10 \mathrm{yr})$ & $17 / 12$ & $13 / 10$ & 0.550 \\
\hline Extent of involvement (pancolitis/left-sided colitis \&t proctitis) & $9 / 20$ & $9 / 14$ & 0.380 \\
\hline Mayo endoscopic subscore (0/1 or 2) & $10 / 19$ & $13 / 10$ & 0.095 \\
\hline Inflammation in terminal ileum (present/absent) & $0 / 29$ & $3 / 20$ & 0.080 \\
\hline Reddish peri-appendiceal mucosa (present/absent) & $2 / 27$ & $8 / 15$ & 0.014 \\
\hline Oral steroid use within 1 year (yes/no) & $4 / 25$ & $4 / 19$ & 0.510 \\
\hline Use of aminosalicylate (yes/no) & $23 / 6$ & $18 / 5$ & 0.930 \\
\hline Use of immunomodulator/anti-TNF antibody/tacrolimus (yes/no) & $3 / 26$ & $0 / 23$ & 0.170 \\
\hline
\end{tabular}

L-type, low-type; H-type, high-type; TNF, tumor necrosis factor.
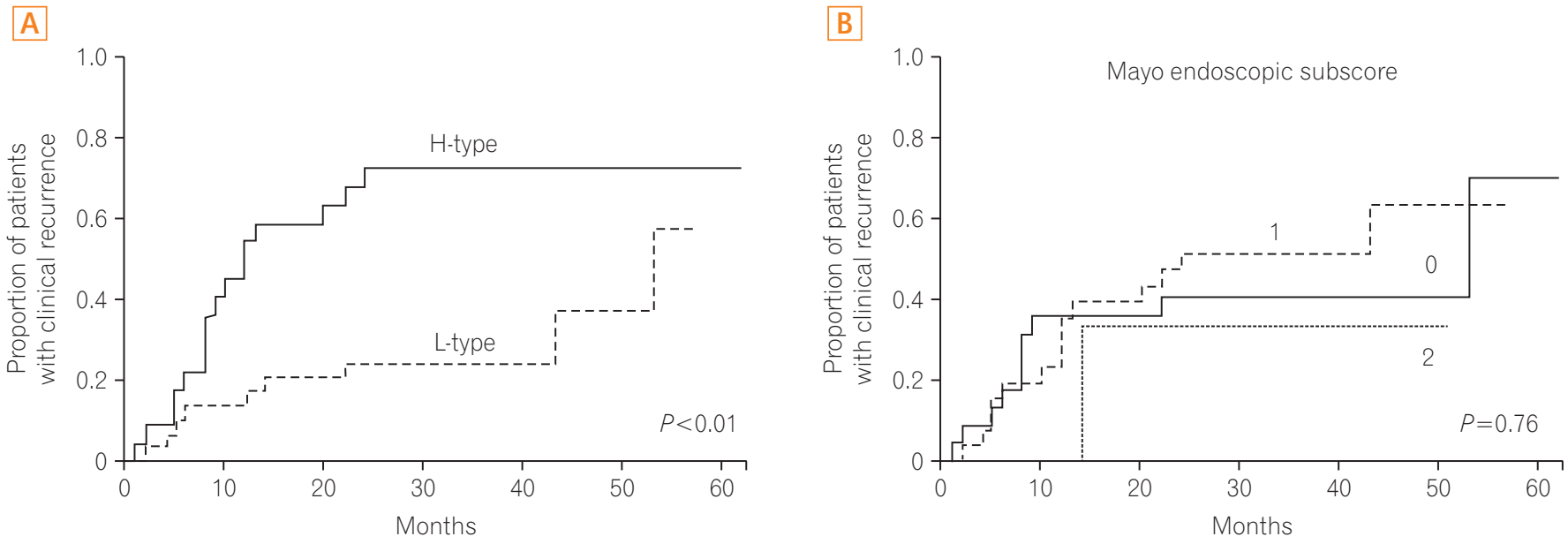

Fig. 3. Results of the Kaplan-Meier survival analysis showing the cumulative proportion of patients who experienced clinical recurrence based on Peyer's patch imaging using narrow-band imaging with magnifying endoscopy (A) and Mayo endoscopic subscore (B). H-type, high-type; L-type, low-type.

rate after colonoscopy is shown in Fig. 3A. Kaplan-Meier survival analysis showed that the clinical recurrence rate was significantly higher in patients with H-type PPs than in those with L-type PPs $(P<0.01)$. The cumulative clinical recurrence rates 12 and 24 months after colonoscopy were 17\% and $25 \%$, respectively, in patients with L-type PPs, whereas they were $54 \%$ and $73 \%$, respectively, in patients with H-type PPs. In contrast, the cumulative clinical recurrence rates did not differ significantly among patients with different Mayo endoscopic subscores $(0,1$, or 2) (Fig. 3B).

\section{Prognostic Factors for Relapse}

We further evaluated the ability of NBI-ME to predict clini- cal relapse in patients with UC. Using univariate analysis, the following factors were found to be significantly associated with relapse: (1) young age at the time of ileocolonoscopy (hazard ratio [HR], 3.0; 95\% CI, 1.3-7.3; $P=0.0090$ ); (2) young age at diagnosis (HR, 2.9; 95\% CI, 1.2-8.2; $P=0.0180)$; (3) oral steroid use in the previous year (HR, 3.3; 95\% CI, 1.2-8.0; $P=0.0230$ ); and (4) H-type PPs (HR, 3.1; 95\% CI, 1.4-7.5; $P=0.0052)$. Neither the extent of colonic involvement nor the Mayo endoscopic subscore were associated with relapse (Table 3). Among the four significant factors mentioned, multivariate analysis showed that H-type PPs were significantly associated with relapse (HR, 3.3; 95\% CI, 1.4-8.2; $P=0.0047$ ) (Table 3). 
Table 3. Predictors of Clinical Recurrence according to Univariate and Multivariate Analyses

\begin{tabular}{|c|c|c|c|c|}
\hline \multirow{2}{*}{ Baseline factor } & \multicolumn{2}{|c|}{ Univariate analysis } & \multicolumn{2}{|c|}{ Multivariate analysis } \\
\hline & $P$-value & $\mathrm{HR}(95 \% \mathrm{Cl})$ & $P$-value & $\mathrm{HR}(95 \% \mathrm{Cl})$ \\
\hline Age (<40 yr) & 0.0090 & $3.00(1.30-7.30)$ & 0.3600 & $1.70(0.58-6.00)$ \\
\hline Age at diagnosis (<40 yr) & 0.0180 & $2.90(1.20-8.20)$ & 0.3500 & $1.90(0.47-7.10)$ \\
\hline Female sex & 0.4700 & $0.74(0.32-1.70)$ & - & - \\
\hline Disease duration (<10 yr) & 0.2900 & $1.60(0.70-3.70)$ & - & - \\
\hline Extent of involvement (pancolitis) & 0.6900 & $1.20(0.50-2.60)$ & - & - \\
\hline Mayo endoscopic subscore (0) & 0.7900 & $0.90(0.39-2.00)$ & - & - \\
\hline Inflammation in terminal ileum (present) & 0.2200 & $2.80(0.45-9.70)$ & - & - \\
\hline Oral steroid use within 1 year (yes) & 0.0230 & $3.30(1.20-8.00)$ & 0.1100 & $2.40(0.81-6.10)$ \\
\hline Use of aminosalicylate (yes) & 0.1900 & $2.00(0.73-6.80)$ & - & - \\
\hline Use of immunomodulator/anti-TNF antibody/tacrolimus (yes) & 0.4600 & $0.51(0.028-2.400)$ & - & - \\
\hline Irregularity of villi (H-type) & 0.0052 & $3.10(1.40-7.50)$ & 0.0047 & $3.30(1.40-8.20)$ \\
\hline
\end{tabular}

HR, hazard ratio; TNF, tumor necrosis factor; H-type, high-type.

\section{DISCUSSION}

This is the first report showing that alterations in the villi of PPs, determined using NBI-ME, are associated with clinical relapse in patients with UC. Several trials have addressed endoscopy, including ME and chromoendoscopy, and shown that the technique is associated with improved assessment of disease activity in patients with UC. ${ }^{11,12}$ For instance, Nishio et al. ${ }^{11}$ reported that an irregular pit pattern, shown using ME and methylene blue staining in the rectal mucosa, is a predictor of relapse, while a study by a Spanish group found that neither chromoendoscopy nor NBI images obtained using rectosigmoidoscopy could predict clinical or endoscopic relapse. ${ }^{12}$ In contrast to both these conflicting sets of data, the present study found that images of PPs obtained using NBI-ME can be used to reliably predict sustained clinical remission.

Only a few reports have addressed the endoscopic appearance of PPs in patients with UC. In one study that involved 18 such patients and used magnifying chromoendoscopy, Ishii et al. ${ }^{13}$ reported that the dome of PPs is irregular, and that it is surrounded by sparse and atrophic villi. In the same study, the association between the endoscopic appearance of PPs and the clinical characteristics of UC, however, was not discussed. In our own study, we used the NBI technique, wherein the vascular network and surface texture of the mucosa can be promptly enhanced, without any additional equipment. ${ }^{14,15}$ Using NBI-ME, we could show that the appearance of PPs may have predictive value for disease process in patients with UC. To further confirm this observation, a prospective study is on-going.

It is urgent that researchers develop biomarkers (ideally non-invasive) to predict the clinical course of UC. ${ }^{16}$ Clinical characteristics, including young age at diagnosis, female gender, or extensive colitis (pancolitis), have been reported as predictors of recurrence in UC. ${ }^{17}$ Although CRP levels do correlate with endoscopic disease activity and clinical relapse in patients with $\mathrm{UC},{ }^{18}$ the sensitivity is poor for predicting relapse in patients with mild disease ${ }^{16}$ Similarly, fecal calprotectin has been shown to be correlated with the clinical activity of UC, and can be useful for predicting clinical relapse.$^{16}$ Indeed, fecal calprotectin can non-invasively detect the intestinal infiltration of neutrophils during mucosal inflammation, before patients become symptomatic. We did not measure fecal calprotectin in the current study, and we need to evaluate the correlation between fecal calprotectin and NBI-ME images in UC. Mucosal healing has been associated with a low risk of hospitalization ${ }^{19}$ and future colectomy ${ }^{20}$ in patients with UC. In this study, we compared patients who had Mayo endoscopic subscores of 0 or 1 with those who had Mayo subscores of 2 (Table 2), because the majority of remissive patients in our cohort had a subscore of 0 or 1 , and because a recent study demonstrated that patients with a Mayo endoscopic subscore of 1 had a higher recurrence rate than those with a subscore of $0{ }^{21}$ In neither group (H- or L-type PPs) was there a significant difference between patients who had a Mayo endoscopic subscore of 0 or 1 and those who had a subscore of 2. Similarly, in our cohort of remissive patients, Mayo endoscopic subscore was not a significant predictor of relapse. In contrast, there was 
statistically significant difference rate between L-type and H-type PPs in terms of recurrence rate. Therefore, the appearance of PPs may be useful in predicting relapse before the patients concerned exhibit intestinal inflammation or elevated fecal calprotectin.

The cause of the alterations to PPs in UC has not been identified. Backwash ileitis is sometimes observed in UC patients who have severe pancolitis; this is caused by an incompetent Bauhin's valve. ${ }^{22}$ However, as we analyzed remissive patients, it is unlikely that the PP alterations had been caused by spreading inflammation, such as backwash ileitis. Interestingly, patients with altered PPs had a significantly higher rate of peri-appendiceal inflammation. Peri-appendiceal lesions in the cecum have been considered distinct "skip lesions" of UC, and have been shown to have prognostic implications for the course of the disease. ${ }^{23}$ In our study, however, the involvement of peri-appendiceal lesions had no predictive value for UC relapse (data not shown). On the same note, a recent report has suggested that cecal patches, the lymphoid clusters that exist in the appendix, also function as a major component of the GALT, similarly to PPs. ${ }^{24}$ Therefore, we need to further clarify the role of the GALT in UC.

This study had several limitations. Firstly, it was a singlecenter study with a relatively small sample size. Moreover, we mainly evaluated patients in remission, and the number of patients with active disease was small. In addition, the influence of treatments for UC on the imaging was not analyzed sufficiently. Therefore, it will be necessary to carry out further investigation into PPs by using NBI-ME in patients with various disease types; long-term follow-up will also be required. To further clarify the role of PPs in UC, a multicenter study is currently underway.

In conclusion, using NBI-ME to observe PPs may be useful in predicting the clinical course of UC; specifically, UC patients with morphological PP alterations showed recurrence. Further studies are required to determine the relationship between the changes in PPs and the disease course of UC.

\section{REFERENCES}

1. Iijima H, Takahashi I, Kiyono H. Mucosal immune network in the gut for the control of infectious diseases. Rev Med Virol 2001;11:117-133.

2. Van Kruiningen HJ, West AB, Freda BJ, Holmes KA. Distribution of Peyer's patches in the distal ileum. Inflamm Bowel Dis 2002;8:180-185.
3. Leone V, Chang EB, Devkota S. Diet, microbes, and host genetics: the perfect storm in inflammatory bowel diseases. J Gastroenterol 2013;48:315-321.

4. MacDonald TT, Spencer J, Viney JL, Williams CB, Walker-Smith JA. Selective biopsy of human Peyer's patches during ileal endoscopy. Gastroenterology 1987;93:1356-1362.

5. Annese V, Daperno M, Rutter MD, et al. European evidence basedconsensus for endoscopy in inflammatory bowel disease. J Crohns Colitis 2013;7:982-1018.

6. Hiyama S, Iijima H, Shinzaki S, et al. Narrow band imaging with magnifying endoscopy for Peyer's patches in patients with inflammatory bowel disease. Digestion 2013;87:269-280.

7. Podolsky DK. Inflammatory bowel disease (1). N Engl J Med 1991;325:928-937.

8. Podolsky DK. Inflammatory bowel disease (2). N Engl J Med 1991;325:1008-1016.

9. Schroeder KW, Tremaine WJ, Ilstrup DM. Coated oral 5-aminosalicylic acid therapy for mildly to moderately active ulcerative colitis: a randomized study. N Engl J Med 1987;317:1625-1629.

10. Cornes JS. Number, size, and distribution of Peyer's patches in the human small intestine. Part I: the development of Peyer's patches. Gut 1965;6:225-229.

11. Nishio Y, Ando T, Maeda O, et al. Pit patterns in rectal mucosa assessed by magnifying colonoscope are predictive of relapse in patients with quiescent ulcerative colitis. Gut 2006;55:17681773.

12. Jauregui-Amezaga A, López-Cerón M, Aceituno M, et al. Accuracy of advanced endoscopy and fecal calprotectin for prediction of relapse in ulcerative colitis: a prospective study. Inflamm Bowel Dis 2014;20:1187-1193.

13. Ishii $H$, Isomoto $H$, Shikuwa $S$, et al. Peyer's patches in the terminal ileum in ulcerative colitis: magnifying endoscopic findings. J Clin Biochem Nutr 2010;46:111-118.

14. Gono K, Obi T, Yamaguchi M, et al. Appearance of enhanced tissue features in narrow-band endoscopic imaging. J Biomed Opt 2004;9:568-577.

15. Kashida H. Usage of narrow band imaging system in the colorectum. Intest Res 2009;7:1-7.

16. Sands BE. Biomarkers of inflammation in inflammatory bowel disease. Gastroenterology 2015;149:1275-1285.e2.

17. Liverani E, Scaioli E, Digby RJ, Bellanova M, Belluzzi A. How to predict clinical relapse in inflammatory bowel disease patients. World J Gastroenterol 2016;22:1017-1033.

18. Henriksen M, Jahnsen J, Lygren I, et al. C-reactive protein: a predictive factor and marker of inflammation in inflammatory bowel disease. Results from a prospective population-based study. Gut 2008;57:1518-1523. 
19. Ardizzone S, Maconi G, Russo A, Imbesi V, Colombo E, Bianchi Porro G. Randomised controlled trial of azathioprine and 5 -aminosalicylic acid for treatment of steroid dependent ulcerative colitis. Gut 2006;55:47-53.

20. Colombel JF, Rutgeerts P, Reinisch W, et al. Early mucosal healing with infliximab is associated with improved longterm clinical outcomes in ulcerative colitis. Gastroenterology 2011;141:1194-1201.

21. Boal Carvalho P, Dias de Castro F, Rosa B, Moreira MJ, Cotter J. Mucosal healing in ulcerative colitis: when zero is better. J Crohns Colitis 2016;10:20-25.
22. Haskell H, Andrews CW Jr, Reddy SI, et al. Pathologic features and clinical significance of "backwash" ileitis in ulcerative colitis. Am J Surg Pathol 2005;29:1472-1481.

23. Park SH, Loftus EV Jr, Yang SK. Appendiceal skip inflammation and ulcerative colitis. Dig Dis Sci 2014;59:2050-2057.

24. Masahata K, Umemoto E, Kayama H, et al. Generation of colonic IgA-secreting cells in the caecal patch. Nat Commun 2014;5:3704. 